\title{
Satellite Spectral Property Observations in Chilean Lakes
}

\author{
Patricio De los Ríos Escalante ${ }^{1,2 *}$, Manuel Castro ${ }^{3}$, \\ Carlos Esse ${ }^{1,2}$, Patricio Acevedo ${ }^{3}$ \\ ${ }^{1}$ Laboratorio de Ecología Aplicada y Biodiversidad, Escuela de Ciencias Ambientales, \\ Facultad de Recursos Naturales, Universidad Católica de Temuco, Casilla15-D, Temuco, Chile \\ ${ }^{2}$ Núcleo de Estudios Ambientales, UCTemuco \\ ${ }^{3}$ Laboratorio de Teledetección Satelital, Departamento de Ciencias Físicas, \\ Facultad de Ingeniería y Ciencias, Universidad de LaFrontera, Casilla 54-D, Temuco
}

Received: 29 April 2016

Accepted: 18 July 2016

\begin{abstract}
Northern Chilean Patagonia has numerous pristine lakes associated with Nothofagus and Araucaria araucana forests. The present study looks at optical properties in visible, close, and medium infrared wavelengths in mountain lakes located in Huerquehue National Park. These lakes have associated Nothofagus and Araucaria araucana native forests with glacial origin. The satellite results revealed high reflectance values in Los Patos Lagoon to B5, B6, and B7 Landsat-8 satellite bands. This lagoon is the most ephemeral pool located within A. araucana forest, whereas Tinquilco Lake has B5, B6, and B7 low reflectance values, which would be due to the presence of Nothofagus forest and probable human intervention in its surrounding basin. Whereas Angelica, Del Sacrificio, Chico, Los Condores, Las Mercedes, Olvidada, Toro, and San Manuel lagoons have low B3, B4, B5, B6, and B7 reflectance values, which would be because of the presence of native forest in their surrounding basin, and that all of these ecosystems are permanent. These differences, in spite of the oligotrophy, would be associated with surrounding vegetation and geological characteristics of studied sites.
\end{abstract}

Keywords: remote sensing, satellite images, lakes, oligotrophy

\section{Introduction}

The mountain lakes of the Chilean Araucanian Andes are oligotrophic, of glacial or volcanic origin, and are associated with native Nothofagus Blume forest, particularly N. antarctica (G. Forst.) Oerst., N. pumilio (Poepp. et Endl.) Krasser, and N. dombeyi (Mirb.) Oerst.

*e-mail: prios@uct.cl
At altitudes greater than 1,000 m.a.s.l., these species coexist with Araucaria araucana (Molina) K. Koch, between $38-39^{\circ} \mathrm{S}$ [1-3]. South of $39^{\circ} \mathrm{S}$, Nothofagus species predominate, and south of $41^{\circ} \mathrm{S}$ and at altitudes above $1,100 \mathrm{~m}$, shrubs and grass vegetation predominate [2-3]. The environmental heterogeneity of Patagonian lakes has been described in detail mainly regarding trophic status and associated basins [4-6], but recently we studied some optical properties associated with ecological implications due to the presence of associated glaciers with consequent changes in water coloration properties, light absorption, 
and changes in associated trophic webs [7-9], and these results would be associated with optical properties obtained from satellite images [10-12].

The aim of the present study is to compare spectral property data obtained from LANDSAT-8/OLI in lakes located in lagoons within Huerquehue National Park, which is a mountain zone with native forest of Nothofagus at low altitudes, that is replaced gradually by A. araucana and shrubs at high altitudes and with many lakes associated with different surrounding vegetation $[1,3]$. These sites are located within a mountain zone with different surrounding landscapes, from Nothofagus forests and shrubs in Tinquilco lake, and Nothofagus and A. araucana forests for Los Condores, Olvidada, Chico, and Del Sacrificio lagoons. Los Patos lagoon is located inside $A$. araucana forest with shrubs, and Las Mercedes and San Manuel lagoons are located in a shrub zone with presence of A. araucana. Many of these lakes are located in zones with serious access problems, and only a few of them are not accessible by mountain paths [1, 3]. Many of these lakes are located in zones without easy access, and in this scenario the first exploration by remote sensing techniques would be very useful for begin limnological studies [9, 13-15].

\section{Material and Methods}

The remote sensing procedures use a Landsat- 8 operational land imager (OLI) image from 31 January 2016. The Landsat- 8 image is provided by the Land Processess Distributed Active Archive Center (LP-DAAC) of the U.S. Geological Survey (LPDAAC.usgs.gov). The spectral and spatial characteristics of the OLI sensor are presented in Fig. 1 and Table 1. The bands of visible, near, and mid-infrared were calibrated radiometrically to spectral radiance and then to reflectance with atmospheric correction being applied.

This image is used to obtain data of different lakes and lagoons within Huerquehue National Park (Fig. 2). The obtained reflectance of Huerquehue National Park lakes of OLI sensor are presented in Table 2. Reflectance data analysis was applied as a principal correspondence analysis to obtain the grouping for sampled sites. This

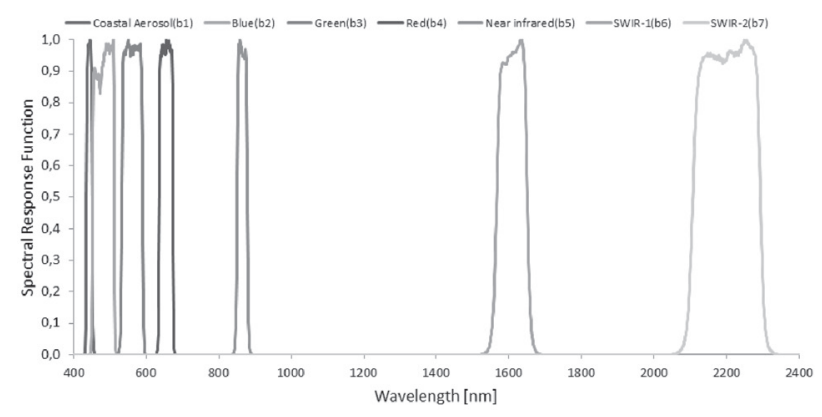

Fig. 1. Relative spectral response: Landsat-8/OLI sensor reflective bands (1-7).
Table 1. Technical characteristics of the Landsat-8/OLI sensor reflective bands.

\begin{tabular}{|c|c|c|c|c|}
\hline Band & $\begin{array}{c}\text { Spectral } \\
\text { Range [nm] }\end{array}$ & $\begin{array}{c}\text { Wavelength } \\
\text { Center [nm] }\end{array}$ & GSD[m] & $\begin{array}{c}\text { Band } \\
\text { Name }\end{array}$ \\
\hline 1 & $430-450$ & 443 & 30 & $\begin{array}{c}\text { Coastal/ } \\
\text { Aerosol }\end{array}$ \\
\hline 2 & $450-510$ & 482 & 30 & Blue \\
\hline 3 & $530-590$ & 562 & 30 & Green \\
\hline 4 & $640-670$ & 655 & 30 & Red \\
\hline 5 & $850-880$ & 865 & 30 & NIR \\
\hline 6 & $1,570-1,650$ & 1,610 & 30 & SWIR1 \\
\hline 7 & $2,110-2,290$ & 2,200 & 30 & SWIR2 \\
\hline
\end{tabular}

statistical analysis was applied using the software R [16] and the Package HSAUR [17], based on methodology used for Patagonian lakes [10-12].

Additionally, two other Landsat- 8 images were used (dated 25 January 2014 and 28 January 2015) to confirm whether the results have the consistency and reproducibility of other days. In this case dates for the summer period are compared in order to eliminate the effects of phenological changes in vegetation surrounding lakes and lagoons.

\section{Results and Discussion}

The correlation analysis (Pearson correlation test) revealed only direct significant correlations between $\mathrm{B} 1$ with $\mathrm{B} 2\left(\mathrm{R}^{2}=0.912 ; \mathrm{p}<0.05\right), \mathrm{B} 2$ with $\mathrm{B} 3\left(\mathrm{R}^{2}=0.714\right.$; $\mathrm{p}<0.05)$, B3 with B4 $\left(\mathrm{R}^{2}=0.894 ; \mathrm{p}<0.05\right)$, B4 with B5 $\left(\mathrm{R}^{2}=0.691 ; \mathrm{p}<0.05\right)$, B4 with $\mathrm{B} 6\left(\mathrm{R}^{2}=0.690\right.$; $\mathrm{p}<0.05), \mathrm{B} 4$ with $\mathrm{B} 7\left(\mathrm{R}^{2}=0.719 ; \mathrm{p}<0.05\right), \mathrm{B} 5$ with $\mathrm{B} 6$ $\left(\mathrm{R}^{2}=0.995 ; \mathrm{p}<0.05\right)$, B5 with $\mathrm{B} 7\left(\mathrm{R}^{2}=0.983 ; \mathrm{p}<0.05\right)$, and B6 with B7 $\left(\mathrm{R}^{2}=0.995 ; \mathrm{p}<0.05\right)$ (Table 3$)$. The PCA revealed that variables that contributed to axis 1 were $B 4$, $\mathrm{B} 5$, and $\mathrm{B} 7$, whereas $\mathrm{B} 1, \mathrm{~B} 2$, and $\mathrm{B} 3$ contributed to axis 2 (Table 4, Fig. 3).

The results of PCA revealed the existence of a first group joined by Angelica, Chico, Del Sacrificio, Toro, Olvidada, San Manuel, and Los Condores lagoons with low B3, B4, B4, B5, B6, and B7 reflectance values, relatively low altitude, and Nothofagus and A. Araucana forests; also, it denoted a second group joined by Las Mercedes, Huerquehue lagoons, and Verde lake with high $\mathrm{B} 1, \mathrm{~B} 2$, and $\mathrm{B} 3$ reflectance values, relatively high altitude, with $A$. Araucana forests. In addition, Tinquilco Lake has low B5, B6, and B7 reflectance, low altitude, and Nothofagus forests. Finally Los Patos lagoon has high B5, B6, and B7 reflectance value with relatively high altitude, with A. Araucana forests (Fig. 3). The analysis of two other Landsat-8 images used (25 January 2014 and 28 January 2015) confirm the consistency of these results (Tables 5 and 6).

The present study revealed differences in optical properties for studied lakes that could be denoted using 


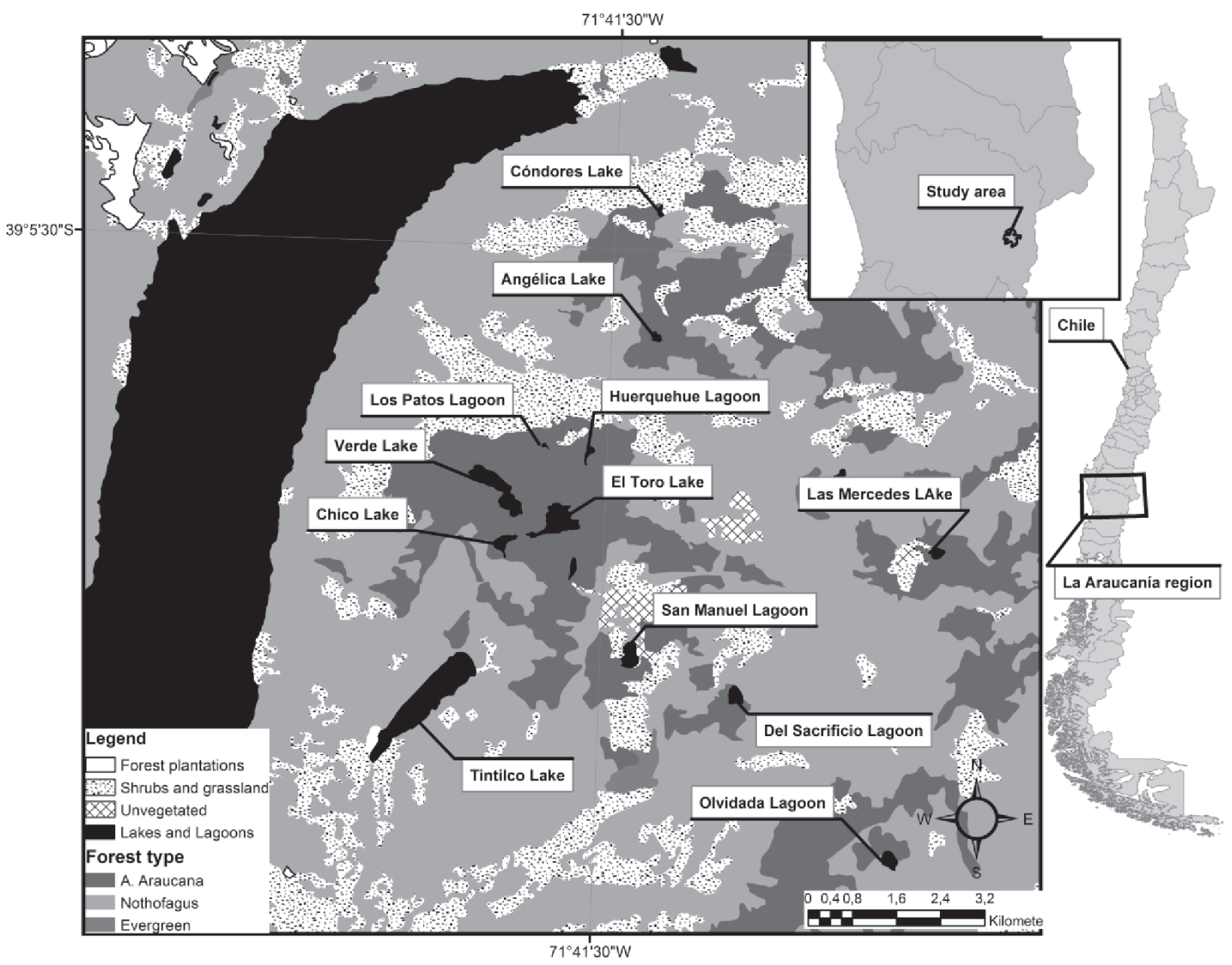

Fig. 2. Study area of Huerquehue National Park.

Table 2. Geographical location, altitude, and reflectance for studied lakes (2016).

\begin{tabular}{|c|c|c|c|c|c|c|c|c|c|}
\hline & Geographical location & $\begin{array}{c}\text { Altitude } \\
\text { (m.a.s.1.) }\end{array}$ & B1 & B2 & B3 & B4 & B5 & B6 & B7 \\
\hline Angelica & $39.1060 \mathrm{~S} ; 71.6823 \mathrm{~W}$ & 1,295 & 0.0147 & 0.0134 & 0.0138 & 0.0114 & 0.0147 & 0.0133 & 0.0116 \\
\hline Chico & $39.1402 \mathrm{~S} ; 71.7127 \mathrm{~W}$ & 1,250 & 0.0153 & 0.0137 & 0.0125 & 0.0107 & 0.0139 & 0.0118 & 0.0109 \\
\hline Del Sacrificio & $39.1639 \mathrm{~S} ; 71.6628 \mathrm{~W}$ & 1,356 & 0.0184 & 0.0169 & 0.0125 & 0.0103 & 0.0136 & 0.0130 & 0.0121 \\
\hline Huerquehue & $39.1252 \mathrm{~S} ; 71.6957 \mathrm{~W}$ & 1,411 & 0.0185 & 0.0199 & 0.0316 & 0.0193 & 0.0179 & 0.0144 & 0.0130 \\
\hline Las Mercedes & $39.1395 \mathrm{~S} ; 71.6217 \mathrm{~W}$ & 1,500 & 0.0208 & 0.0225 & 0.0313 & 0.0225 & 0.0198 & 0.0172 & 0.0155 \\
\hline Los Condores & $39.0853 \mathrm{~S} ; 71.6835 \mathrm{~W}$ & 1,429 & 0.0162 & 0.0151 & 0.0156 & 0.0147 & 0.0195 & 0.0168 & 0.0153 \\
\hline Los Patos & $39.1242 \mathrm{~S} ; 71.7046 \mathrm{~W}$ & 1,466 & 0.0139 & 0.0134 & 0.0202 & 0.0211 & 0.0444 & 0.0334 & 0.0255 \\
\hline Olvidada & $39.1899 \mathrm{~S} ; 71.6294 \mathrm{~W}$ & 1,442 & 0.0157 & 0.0152 & 0.0123 & 0.0100 & 0.0134 & 0.0119 & 0.0111 \\
\hline San Manuel & $39.1580 \mathrm{~S} ; 71.6853 \mathrm{~W}$ & 1,498 & 0.0193 & 0.0175 & 0.0125 & 0.0109 & 0.0138 & 0.0134 & 0.0127 \\
\hline Tinquilco & $39.1667 \mathrm{~S} ; 71.7280 \mathrm{~W}$ & 769 & 0.0196 & 0.0171 & 0.0140 & 0.0113 & 0.0123 & 0.0108 & 0.0105 \\
\hline Toro & $39.1365 \mathrm{~S} ; 71.7012 \mathrm{~W}$ & 1,260 & 0.0166 & 0.0150 & 0.0127 & 0.0109 & 0.0127 & 0.0119 & 0.0115 \\
\hline Verde & $39.1314 \mathrm{~S} ; 71.7137 \mathrm{~W}$ & 1,285 & 0.0205 & 0.0204 & 0.0202 & 0.0148 & 0.0159 & 0.0148 & 0.0138 \\
\hline
\end{tabular}


Table 3. Correlation matrix for variables considered in the present study (values in bold denotes significant correlation; $\mathrm{p}<0.05)$.

\begin{tabular}{|c|c|c|c|c|c|c|c|}
\hline & Altitude & B1 & B2 & B3 & B4 & B5 & B6 \\
\hline B7 & 0.421 & 0.288 & 0.093 & 0.357 & $\mathbf{0 . 7 1 9}$ & $\mathbf{0 . 9 8 3}$ & $\mathbf{0 . 9 9 5}$ \\
\hline B6 & 0.396 & -0.356 & 0.160 & 0.324 & $\mathbf{0 . 6 9 0}$ & $\mathbf{0 . 9 9 5}$ & \\
\hline B5 & 0.354 & 0.395 & 0.190 & 0.335 & $\mathbf{0 . 6 9 1}$ & & \\
\hline B4 & 0.393 & 0.164 & 0.474 & $\mathbf{0 . 8 9 4}$ & & & \\
\hline B3 & 0.310 & 0.405 & $\mathbf{0 . 7 1 4}$ & & & & \\
\hline B2 & 0.107 & $\mathbf{0 . 9 1 2}$ & & & & & \\
\hline B1 & -0.161 & & & & & & \\
\hline
\end{tabular}

Table 4. PCA contribution percentage of variables for axes 1 and 2.

\begin{tabular}{|c|c|c|}
\hline & 1 & 2 \\
\hline Altitude & 0.263 & -0.012 \\
\hline B1 & -0.090 & -0.555 \\
\hline B2 & 0.051 & -0.601 \\
\hline B3 & 0.299 & 0.430 \\
\hline B4 & 0.432 & 0.255 \\
\hline B6 & 0.459 & 0.182 \\
\hline B5 & 0.462 & 0.169 \\
\hline B7 & 0.467 & 0.131 \\
\hline
\end{tabular}

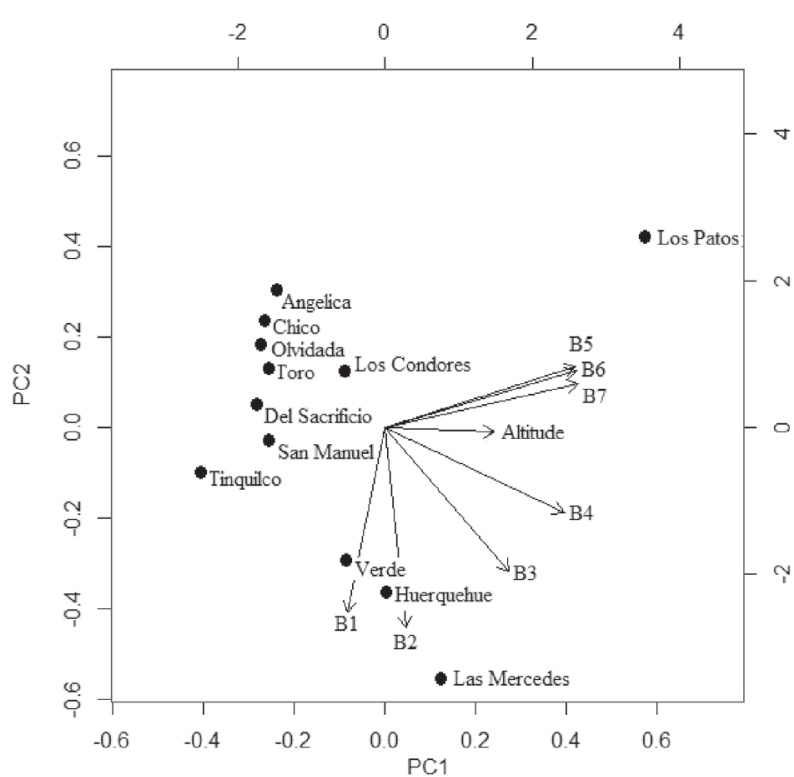

Fig. 3. PCA analysis for variables considered in the present study.

remote sensing techniques such as were observed for Tagua Tagua and General Carrera Patagonian lakes that have marked environmental heterogeneity [10-12].

\begin{tabular}{|c|c|c|c|c|c|c|c|c|c|c|c|}
\hline$\hat{n}$ & 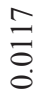 & $\begin{array}{l}\stackrel{ }{\Xi} \\
\stackrel{0}{\circ}\end{array}$ & $\begin{array}{l}\stackrel{0}{\exists} \\
\stackrel{0}{0} \\
\stackrel{0}{0}\end{array}$ & $\begin{array}{l}\vec{\sim} \\
\stackrel{0}{0} \\
\stackrel{0}{0}\end{array}$ & $\begin{array}{l}\stackrel{\sigma}{\Xi} \\
\overline{0} \\
\dot{0}\end{array}$ & $\begin{array}{c}\stackrel{2}{2} \\
\stackrel{0}{0} \\
\stackrel{0}{0}\end{array}$ & $\begin{array}{l}\stackrel{\partial}{J} \\
\stackrel{\partial}{0} \\
\dot{0}\end{array}$ & $\begin{array}{l}\stackrel{\circ}{\circ} \\
\stackrel{0}{0} \\
\dot{0}\end{array}$ & $\begin{array}{l}\cong \\
\stackrel{0}{0} \\
\stackrel{0}{0}\end{array}$ & 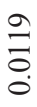 & $\begin{array}{l}\widehat{N} \\
\stackrel{0}{0} \\
\stackrel{0}{0}\end{array}$ \\
\hline 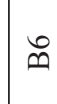 & $\begin{array}{l}\text { Iิ } \\
\text { o. } \\
\stackrel{0}{0}\end{array}$ & $\begin{array}{l}\infty \\
\stackrel{\infty}{0} \\
\stackrel{0}{0}\end{array}$ & 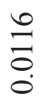 & $\begin{array}{l}\stackrel{+}{m} \\
\stackrel{0}{0}\end{array}$ & $\begin{array}{l}\stackrel{0}{ల} \\
\stackrel{0}{0} \\
0\end{array}$ & $\begin{array}{l}\stackrel{n}{m} \\
\stackrel{0}{0} \\
0\end{array}$ & $\begin{array}{l}\bar{\sigma} \\
\stackrel{0}{0}\end{array}$ & $\begin{array}{l}\text { I } \\
\stackrel{0}{0}\end{array}$ & 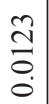 & $\begin{array}{l}\stackrel{ \pm}{I} \\
\stackrel{0}{0}\end{array}$ & $\stackrel{n}{\tilde{n}}$ \\
\hline
\end{tabular}

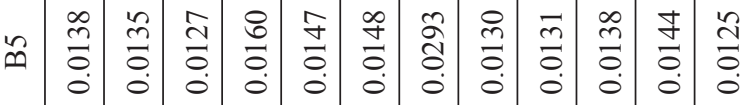

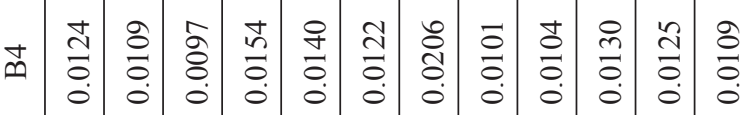

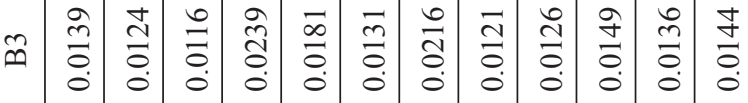

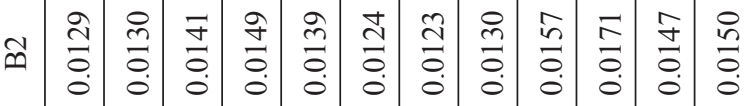

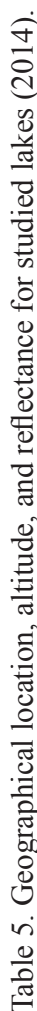

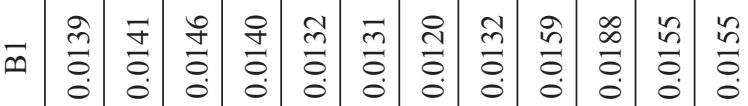

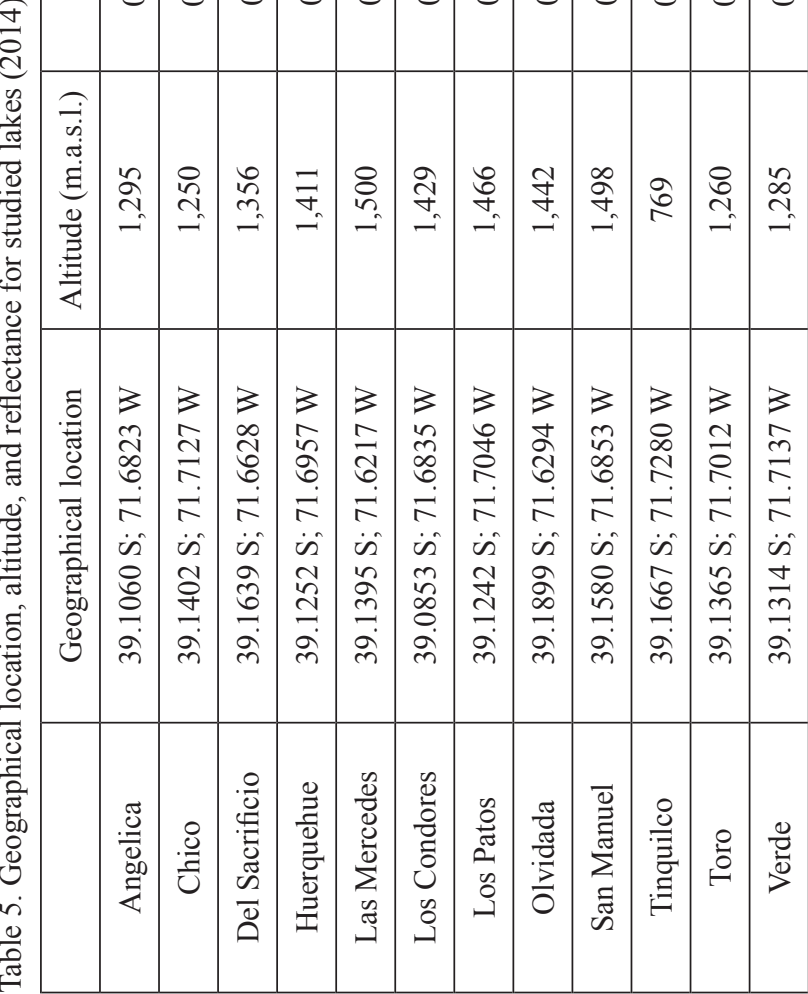




\begin{tabular}{|c|c|c|c|c|c|c|c|c|c|c|c|c|}
\hline 闩 & $\begin{array}{l}\Xi \\
\overrightarrow{0} \\
0 \\
0\end{array}$ & $\begin{array}{l}\infty \\
0 \\
0 \\
0 \\
0\end{array}$ & $\mid \begin{array}{l}0 \\
0 \\
0 \\
0\end{array}$ & $\begin{array}{l}\Omega \\
\bar{a} \\
0 \\
0\end{array}$ & $\mid \begin{array}{l}\vec{J} \\
\bar{\sigma} \\
\dot{0}\end{array}$ & $\begin{array}{l}0 \\
\check{n} \\
0 \\
0\end{array}$ & $\begin{array}{l}\infty \\
\hat{a} \\
0 \\
\dot{0}\end{array}$ & $\begin{array}{l}\stackrel{0}{\overrightarrow{0}} \\
\dot{0} \\
\dot{0}\end{array}$ & $\begin{array}{l}I \\
\Xi \\
0 \\
0\end{array}$ & $\begin{array}{l}\infty \\
0 \\
0 \\
0 \\
0\end{array}$ & 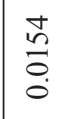 & $\begin{array}{l}0 \\
0 \\
0 \\
0\end{array}$ \\
\hline$\stackrel{\infty}{\infty}$ & $\begin{array}{l}\grave{\Xi} \\
\stackrel{0}{0}\end{array}$ & $\begin{array}{l}0 \\
\vdots \\
0 \\
0\end{array}$ & $\begin{array}{l}I \\
\vdots \\
0\end{array}$ & $\begin{array}{l}\tilde{D} \\
0 \\
0 \\
0\end{array}$ & $\begin{array}{l}0 \\
\overrightarrow{0} \\
\dot{0}\end{array}$ & $\begin{array}{l}\frac{j}{5} \\
0 \\
0\end{array}$ & \begin{tabular}{l}
$n$ \\
\multirow{q}{0}{} \\
0 \\
0
\end{tabular} & $\begin{array}{l}\infty \\
\mathbb{1} \\
0 \\
0\end{array}$ & $\begin{array}{l}0 \\
\vdots \\
0 \\
0\end{array}$ & $\begin{array}{l}m \\
\vdots \\
0\end{array}$ & $\begin{array}{l}0 \\
0 \\
0 \\
0\end{array}$ & $\stackrel{\circ}{\Xi}$ \\
\hline$\ddot{n}$ & $\begin{array}{l}f \\
\stackrel{f}{0} \\
0\end{array}$ & $\begin{array}{l}\text { సे } \\
\stackrel{0}{0}\end{array}$ & $\begin{array}{l}2 \\
\tilde{a} \\
0\end{array}$ & $\begin{array}{l}\Omega \\
0 \\
0 \\
0\end{array}$ & $\begin{array}{l}n \\
0 \\
0 \\
0\end{array}$ & $\begin{array}{l}\overrightarrow{0} \\
0 \\
0 \\
0\end{array}$ & $\begin{array}{l}\tilde{N} \\
\hat{0} \\
0\end{array}$ & $\begin{array}{l}\frac{T}{0} \\
0 \\
0\end{array}$ & $\begin{array}{l}\stackrel{ \pm}{\Xi} \\
\stackrel{0}{0}\end{array}$ & $\begin{array}{l}0 \\
0 \\
0 \\
0 \\
0\end{array}$ & 立 & 离 \\
\hline$\vec{m}$ & $\begin{array}{l}\stackrel{\vartheta}{\overrightarrow{0}} \\
\stackrel{0}{0}\end{array}$ & $\begin{array}{l}8 \\
\stackrel{0}{0} \\
\stackrel{0}{0}\end{array}$ & $\begin{array}{l}\tilde{a} \\
\hat{\sigma} \\
0 \\
0\end{array}$ & $\mid \begin{array}{l}\tilde{\vec{J}} \\
\overrightarrow{0} \\
\dot{0}\end{array}$ & $\mid \begin{array}{l}2 \\
\tilde{a} \\
0 \\
0\end{array}$ & $\begin{array}{l}0 \\
0 \\
0 \\
0\end{array}$ & $\begin{array}{l}\hat{\sigma} \\
\tilde{o} \\
0\end{array}$ & $\begin{array}{l}0 \\
0 \\
0 \\
0\end{array}$ & $\begin{array}{l}0 \\
8 \\
0 \\
0\end{array}$ & $\begin{array}{l}\overrightarrow{\vec{J}} \\
\dot{0}\end{array}$ & $\begin{array}{l}n \\
a \\
0 \\
0\end{array}$ & $\begin{array}{l}n \\
0 \\
0 \\
0\end{array}$ \\
\hline$\tilde{m}$ & $\begin{array}{l}\stackrel{\infty}{d} \\
\stackrel{0}{0} \\
0\end{array}$ & $\begin{array}{l}\infty \\
\stackrel{\mathbb{a}}{0} \\
\stackrel{0}{0}\end{array}$ & $\begin{array}{l}\tilde{y} \\
\bar{a} \\
\dot{0}\end{array}$ & $\begin{array}{l}\infty \\
\vec{\sigma} \\
\dot{\sigma} \\
\dot{0}\end{array}$ & $\begin{array}{l}\tilde{\infty} \\
0 \\
0 \\
0\end{array}$ & $\begin{array}{l}\tilde{n} \\
\vdots \\
0 \\
0\end{array}$ & $\begin{array}{l}\text { } \\
\stackrel{\leftrightarrow}{0} \\
\dot{0}\end{array}$ & $\begin{array}{l}0 \\
\stackrel{n}{0} \\
0 \\
0\end{array}$ & $\begin{array}{l}\infty \\
\stackrel{0}{\Xi} \\
0 \\
0\end{array}$ & $\begin{array}{l}n \\
n \\
0 \\
0 \\
0\end{array}$ & $\frac{0}{0}$ & 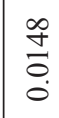 \\
\hline$\tilde{\infty}$ & $\begin{array}{l} \pm \\
\stackrel{0}{0} \\
0 \\
0\end{array}$ & 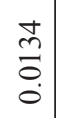 & $\begin{array}{l} \pm \\
0 \\
0 \\
0 \\
0\end{array}$ & $\mid \begin{array}{l}\overrightarrow{\hat{\sigma}} \\
\dot{0} \\
\dot{0}\end{array}$ & 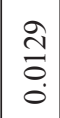 & $\left|\begin{array}{c}\mathcal{J} \\
0 \\
0\end{array}\right|$ & $\begin{array}{l}\tilde{b} \\
\stackrel{0}{0} \\
0\end{array}$ & $\begin{array}{l}n \\
i n \\
0 \\
0\end{array}$ & $\begin{array}{l}0 \\
0 \\
0 \\
0\end{array}$ & $\begin{array}{l}0 \\
0 \\
0 \\
0\end{array}$ & $\begin{array}{l}\widehat{\infty} \\
0 \\
0 \\
0\end{array}$ & $\begin{array}{l}\vec{\sigma} \\
0 \\
0 \\
0\end{array}$ \\
\hline$\vec{m}$ & $\begin{array}{c}\stackrel{m}{0} \\
0 \\
0\end{array}$ & $\begin{array}{l}\frac{J}{d} \\
\dot{0}\end{array}$ & $\begin{array}{l}n \\
0 \\
0 \\
0\end{array}$ & $\mid \begin{array}{c}0 \\
0 \\
0 \\
0\end{array}$ & $\begin{array}{l}\stackrel{2}{0} \\
0 \\
0\end{array}$ & $\left|\begin{array}{c}\hat{y} \\
\dot{0} \\
\dot{0}\end{array}\right|$ & $\begin{array}{l}\stackrel{a}{d} \\
\dot{0}\end{array}$ & $\begin{array}{l}\infty \\
\stackrel{2}{a} \\
0 \\
0\end{array}$ & $\begin{array}{l}\tilde{\infty} \\
\stackrel{0}{0} \\
\dot{0}\end{array}$ & $\begin{array}{l}\sigma \\
\sigma \\
0 \\
0\end{array}$ & $\begin{array}{l}\vec{\sigma} \\
\overrightarrow{0} \\
\dot{0}\end{array}$ & $\begin{array}{l}\tilde{6} \\
0 \\
0\end{array}$ \\
\hline 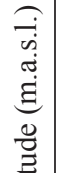 & ڤે & $\begin{array}{l}0 \\
\stackrel{n}{-} \\
\rightarrow\end{array}$ & $\begin{array}{l}\stackrel{2}{\rightleftharpoons} \\
\stackrel{2}{n}\end{array}$ & $\underset{\exists}{\exists}$ & $\begin{array}{l}8 \\
\stackrel{n}{-1} \\
-1\end{array}$ & 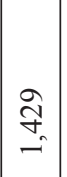 & $\begin{array}{l}0 \\
: \\
\vdots \\
-\end{array}$ & $\underset{\sim}{\mathcal{G}}$ & $\begin{array}{l}\infty \\
\stackrel{a}{\sigma} \\
\underset{-}{+}\end{array}$ & oे & $\begin{array}{l}8 \\
\stackrel{1}{-}\end{array}$ & $\stackrel{\infty}{\infty}$ \\
\hline 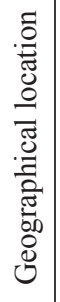 & 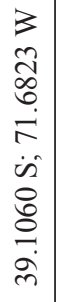 & 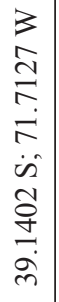 & 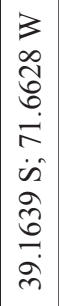 & 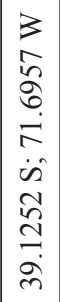 & 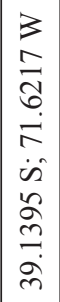 & 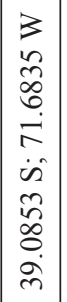 & 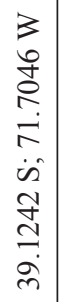 & 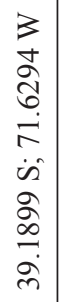 & 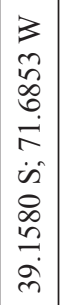 & 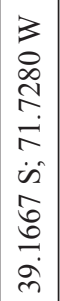 & 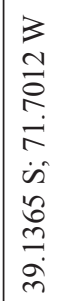 & 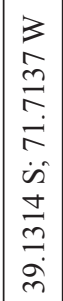 \\
\hline & 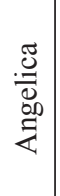 & 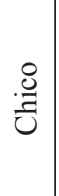 & 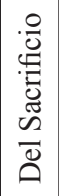 & 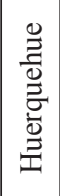 & 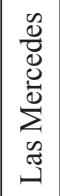 & $\mid \begin{array}{l}0 \\
0 \\
0 \\
0 \\
0 \\
0 \\
0 \\
0 \\
0 \\
0\end{array}$ & $\begin{array}{l}n \\
0 \\
0 \\
0 \\
0 \\
0 \\
0 \\
\ddots\end{array}$ & $\begin{array}{l}\frac{\pi}{\frac{\pi}{\pi}} \\
\frac{\vec{g}}{\vec{D}} \\
0\end{array}$ & 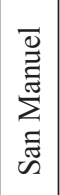 & 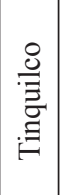 & $\stackrel{\circ}{\circ}$ & $\frac{0}{0}$ \\
\hline
\end{tabular}

In conclusion, it is possible to use satellite reflectance data for monitoring the chemical and trophic status of lakes and lagoons. For example, in the case of Los Patos lagoons, in the three years analyzed, permanently the reflectance values in the infrared bands are superior to the other lakes and lagoons, agreeing that they correspond to more gaps in shallower, smaller, and more ephemeral pools. Also, it is possible to relate high reflectance values to A. Araucana forests, although low reflectance values appear to be related to Nothofagus mixed with $A$. Araucana forests.

The results presented indicate that a potential correlation between environmental associations due to surrounding basins and optical properties might possibly be found; however, it would be necessary to carry out more intensive studies and obtain more data to be able to confirm or discount the possibility of finding potential correlations and their variations at multiple spatial and temporal scales [18-19].

\section{Acknowledgements}

The present study was funded by the TIDES TRF1303011 and MECESUP UCT 0804 projects for their supports in fieldwork. We also thank M.I. for her valuable comments and suggestions.

\section{References}

1. HAUENSTEIN E., BARRIGA K., DE LOS RÍOSESCALANTE P. Macrophytes assemblages in mountain lakes of Huerquehue National Park $\left(39^{\circ} \mathrm{S}\right.$, Araucanía Region, Chile). Latin American Journal of Aquatic Research, 39, 593, 2011.

2. DE LOS RÍOS-ESCALANTE P., HAUENSTEIN E., ACEVEDO P., ROMERO-MIERES M., PANDOURSKI I. Regulatory factors in crustacean zooplankton assemblages in mountain lakes of northern Chilean Patagonia (38-41 $\left.{ }^{\circ} \mathrm{S}\right)$ : a comparison with Bulgarian counterparts. Latin American Journal of Aquatic Research, 40, 473, 2012.

3. DE LOS RÍOS P., HAUENSTEIN E., ACEVEDO P., JAQUE $X$. Littoral crustaceans in mountain lakes of Huerquehue National Park $\left(38^{\circ} \mathrm{S}\right.$, Araucania region, Chile). Crustaceana, 80, 401, 2007.

4. MODENUTTI B.E., BALSEIRO E.G., QUEIMALIÑOS C.P., SUAREZ D.A., DIÉGUEZ M.C. ALBARIÑO R.J. Structure and dynamics of food webs in Andean lakes. Lak. Reserv., Res. Manage., 3, 179, 1998.

5. SOTO, D. Oligotrophic patters in southern Chilean lakes: the relevance of nutrients and mixing depth. Rev. Chil. Hist. Nat., 75: 377, 2002.

6. KROGH S.A., POMEROY J.W., McPHEE J., Physically based mountain hydrological modeling using reanalysis data in Patagonia. Journal of Hydrometeorology, 16: 172, 2015.

7. PASQUINI A. I., DEPETRIS P. J., Southern Patagonia's Perito Moreno Glacier, Lake Argentino and Santa Cruz river hydrological system: an overview. Journal of Hydrology, 405: 48, 2011.

8. LASPOUMADERES C., MODENUTTI B., SOUZA M., BASTIDAS M., CUASSOLO F., BALSEIRO E. Glacier 
melting and stoichiometric implications for lake community structure: zooplankton species distributions across a natural light gradient. Global Change Biology, 19, 316, 2013.

9. HYLANDER S., JEPHSON T., LEBRET, K., VON EINEM J., FAGEBERG T., BALSEIRO E., MODENUTTI B., SOUZA M., LASPOUMADERES, C., JÖHNSON M., LJUNGBERG P., NICOLLE A., NILSSON P.A., RANAKER L., HANSSON L.A. Climate-induced imput of turbid glacial meltwater affects vertical distribution and community composition of phyto- and zooplankton. Journal of Plankton Research, 33, 1239, 2011.

10. DE LOS RÍOS-ESCALANTE P., ACEVEDO P. First observations on zooplankton and optical properties in a glacial north Patagonian lake (Tagua Tagua lake, $41^{\circ} \mathrm{S}$ Chile). Polish Journal of Environmental Studies, 25, 453, 2016.

11. DE LOS RÍOS-ESCALANTE P., ACEVEDO P. First observations of Boeckella michaelseni Mrázek 1901 (Crustacea, Copepoda) and optical properties of central Patagonian lake (General Carrera Lake, $46^{\circ} \mathrm{S}$ Chile). Polish Journal of Environmental Studies, In press.

12. DE LOS RÍOS-ESCALANTE P., QUINAN E., ACEVEDO P. Crustacean zooplankton communities in lake General Carrera $\left(46^{\circ} \mathrm{S}\right)$ and their possible association with optical properties. Crustaceana, 86, 507, 2013.

13. PHAN V.H., LINDENBERGH R., MENENTI M. Seasonal trends in Tibetan lake level changes as observed by icesat laser altimetry. ISPRS Annals of the Photogrammetry, Remote Sensing and Spatial Information Sciences, I-7, 237, 2012.

14. KUTSER T., PAAVEL B., VERPOORTER C., KAUSER T, VAHTMAE E. Remote sensing of water quality in optically complex lakes. ISPRS Annals of the Photogrammetry, Remote Sensing and Spatial Information Sciences, XXXIX-B8, 165, 2012.

15. DE LOS RIOS-ESCALANTE P., PANDOURSKI I., ACEVEDO P. Spectral properties observations first comparison in Bulgarian and Chilean mountain lakes. Polish Journal of Environmental Studies, In press.

16. R DEVELOPMENT CORE TEAM. 2009. R: A language and environment for statistical computing. $\mathrm{R}$ foundation for statistical computing, Vienna, Austria.

17. EVERITT, B.S., HOTHORN, T. A handbook of Statistical Analysis using R ( $1^{\text {st }}$ Edition). https://cran.r-project.org/web/ packages/HSAUR/HSAUR.pdf (Visited $1^{\text {th }}$ Jule 2016).

18. PALMER S.C.J., HUNTER P.D., LANKESTER T., HUBBARD S., SPYRAKOS E., TYLER A.N., PRÉSIG M., HORVATH H., LAMB, A., BALSTER H., TOTH V.R., Validation of envisat MERIS algorithms for chlorophyll retrievan in a large turbid and optically-complex shallow lake. Remote Sensing of Environment 157, 158, 2015.

19. PALMER S.C.J., KUTZER T., HUNTER P.D. Remote sensing of inland waters: challenges, progress and future directions. Remote sensing of Environment, 157,1, 2015. 